\title{
Explosive Detection in El Alamein
}

\author{
Iraky Kalifa \\ Department of Computer \\ Science, Faculty of Computer \\ and Information, Helwan \\ University, Egypt
}

\author{
Aliaa Youssif \\ Department of Computer \\ Science, Faculty of Computer \\ and Information, Helwan \\ University, Egypt
}

\author{
Ahmed Adel \\ Department of Computer \\ Science, Faculty of Computer \\ and Information, Helwan \\ University, Egypt
}

\begin{abstract}
This paper presents an integrated applications and hardware systems of a RC Truck to detect and pinpoint the locations of explosives in desert environment as El Alamein region. This work will be based on the use of the packet oriented mobile data service that available through the global system for mobile communications. By using of this integrated technologies, this paper provides the safe distance to the human in the process of explosives detection and help to map the landmines in fenced minefields and suspicious regions. The truck equipment brought together to fulfill four main tasks, a) Tracing the explosives, b) Detecting the explosives c) Monitor the minor change happens to the explosive detector sensor d) pinpoint the exact location coordinates of those explosives.
\end{abstract}

\section{Keywords}

Detecting Explosives, Suspicious Regions, Tracing Explosives, Coordinates, General Packet Radio Service, Mobile Data Service, Global System For Mobile Communications, Multimedia Messaging Service, Wireless Application Protocol, GSM Sound Tracker Devices, GSM Surveillance Camera, GPS Tracker Devices, Landmines, Metal Detector, Mapping Software and Google Earth.

\section{INTRODUCTION}

Taking care about environment is a recent phenomenon that widely spread in fact after the World War II (WWII) [1]. The development of science and technology leads to fatal environmental problems especially regarding disputes and wars. One of the worst problems that face the humanity is the buried landmines and unexploded ordnance (UXO)[2] due to the long life of these mines, the victims often have no relation to the original motivation for the mines[3], according to the International Campaign to Ban Landmines, they have estimated that $15,000-20,000$ people are injured or killed by landmines each year[4]. [5] point out that these mines kill or injure a person every 20 minutes -70 persons a day, or more than 20,000 people a year. Most victims are children [5] A landmine defines as a device designed to kill or injure anyone that comes in contact with it through direct pressure or a tripwire [6]. Antipersonnel landmines originated in WWII, where Germans and Italians improvised antipersonnel land mines with grenades and fuses in order to prevent allied soldiers from deactivating antitank mines placed on already determined defense lines [7]. Landmines can be categorized into two types: anti-tank (AT) mines and anti-personnel (AP) mines. AT mines are larger, i.e. $20-30 \mathrm{~cm}$ in diameter, whereas AP mines are approximately $5-15 \mathrm{~cm}$ in diameter [8]. In fact, there are more than 350 types of antipersonnel landmines being developed in more than 50 countries [9].
Certain studies point out that there are around 50-100 million AP mines in more than 80 countries around the world. The cost of producing a mine is as little as $\$ 3$, but it can cost as much as $\$ 1000$ to remove it[3],[10] point out that this horizon will retreat further, mainly because of new mines being constantly laid, and also because of the very limited use of technology for mine detection and clearance, and due to the lack of funds for detection. The efforts to establish mono (single) or dual (combined) techniques for landmine detections are growing since the WWII. There are several methods for landmine detection [11] These methods include ground-penetrating radar (GPR), electrical impedance tomography (EIT), x-ray backscatter, and infrared/hyper spectral systems. Different varieties of landmines can be found depending on the casing materials (metallic or plastic), the landmine size and the purpose of using (antipersonnel or antitank) [12], the current conventional tools include in principle metal detector (MD)[13]. In [13] point out that all metallic objects are detected and identified by a metal detector. It is interesting to note that the present paper would introduce a new approach based on metal detector.

\section{SYSTEM DESCRIPTION}

The present paper concentrates on the detection and localization of WWII explosives in El Alamein region by using light, simple and a low cost multi-tasking truck that detect tiny metal objects present in most landmines and localize coordinates. The Explosive Detector Truck (EDT) is a mini RC truck, it is controlled by the human to go all directions from a safe distance in detection and pinpoint processes, The rest of the tracking units and other devices were attached to the truck will be managed by a cell phone that will be used as a Multi-tasking controller by the use of human. In[14] Robot is used in humanitarian demining operation to reduce risks on human operators and to speed up operations. The EDT platform will show in section (3), EDT is caring a three of micro sensitive devices and one light cell phone for tracing explosives, in our case this cell phone is working without SIM Card, it will be used as a sensitive sensor to detect explosives as shown in section (4.1), the rest of all portable unites will be harnessed to perform two deferent major processes, one to monitor any minor change happens to the explosive detector sensor, in our case the GSM surveillance camera and the GSM sound tracer device will replace the human's audiovisual perception system as shown in section (4.2), the second to pinpoint the coordinates of those explosives with the GPS tracker devices as will shown in section (4.3) . 


\section{EDT PLATFORM STRUCTURE DESIGN}

With a closer look to figure 1, will notice The EDT platform, it is made of a light weight plastic and equipped with two DC motors, at the back the conventional two wheels for driving the EDT by 24 Volts DC motor with max 190 rpm speed because high speed is not preferred at detection process and a stepper motor for steering the front two wheels, the steering co-ordinated are actuated using 24 Volts stepper motor with 1.8 degrees per step, while one wooden arm is fixed and extended from the front part of the truck to carry the metal detector sensor to apply the front scanning for explosives, there are different configuration for the arm which is used for the detection, in[15] a 3 DOF is used for full surface contact interfacing, in [16] a 2 DOF used an arm manipulator with two steeper motors for linear and rotational movement. In this paper EDT currently gross weight not exceed 1000 grams, by distributing the total weight on the four wheels that's make the EDT too light to set off mines. [17] point out that HeroRATs are trained to detect and pinpoint the location of a landmine, their size and weight are mostly just over a kilogram this make it highly unlikely that they would set of a pressure-activated mine by scratching or pointing.

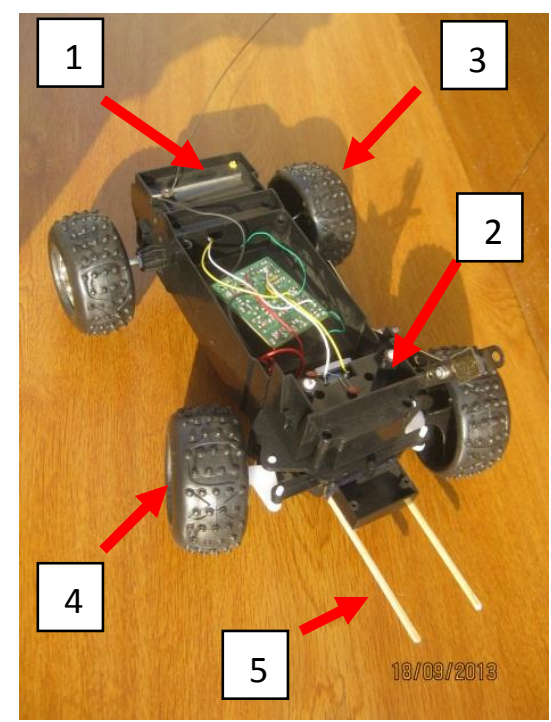

Fig 1: EDT platform structure design. (1) The back motor to control the back wheels, (2) The stepper motor for steering the front two, (3) One of the conventional two wheels, (4) One of the front wheels, (5) The fixed wooden arm to the front of EDT.

\section{SOLVING PROBLEM APPROACH}

\subsection{The explosives tracing strategy}

In [13] point out that all metallic objects are detected and identified by a metal detector, The basic principle on which a metal detector works is that when electric current passes through a coil, it produces a magnetic field around it. A metal detector consists of an oscillator which produces alternating current. When this alternating current passes through the transmit coil present in the metal detector, a magnetic field is produced around it. Whenever an electrically conductive metallic object comes in contact with the coil, it produces another magnetic field around it. The metal detector contains another coil in its loop called receiver coil, which detects the changes in the magnetic field caused due to presence of the metallic object. The current conventional tools include in principle metal detector [13]. From this basis our work has relied on the Metal Detector App for HTC cellphone and a light HTC cellphone unit to act as a sensitive metal detector for tiny metals, a metal detector application downloaded on Android cellphone that were attached to the fixed wooden arm in front of the EDT for detecting process as shown in figure 2, this application acts as a tool for finding metal, by interacting with the magnetic sensor built into the cellphone, it use the magnetometer to measure the magnetic field in the surrounding area, the intensity of the magnetic field in nature is about $49 \mu \mathrm{T}$ or $490 \mathrm{mG}, 1 \mu \mathrm{T}=10 \mathrm{mG}$, as shown in fig 3. If there is any tiny metal (like iron or steel) in the area or buried under the ground's surface, the strength of the magnetic field should increase, which leads to many of changes that showing up on the HTC's cellphone screen as shown in figure 4, to make sure of the sensitivity of HTC metal detector, an explosive capsule has been made manually similar to the form of anti-personnel mines as shown in figure 5.

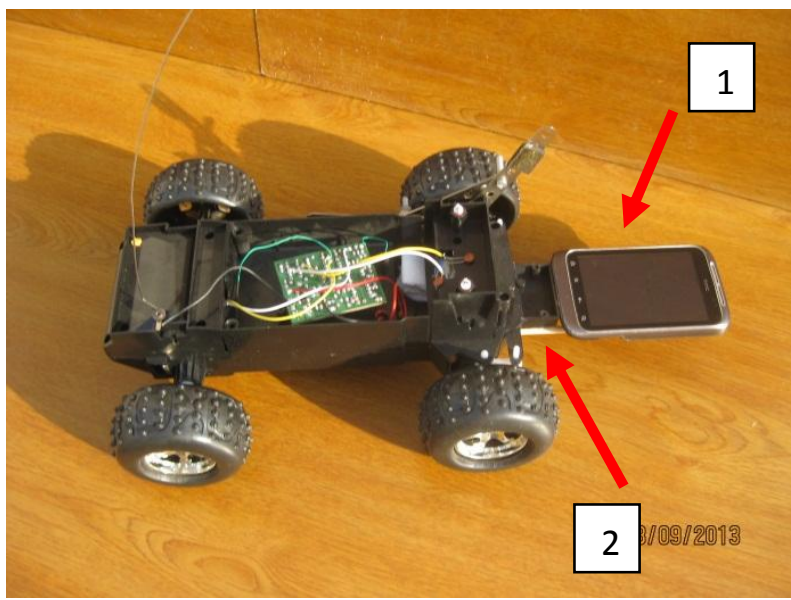

Fig 2: The Android cellphone were put on the wooden arm. (1) Android cellphone for land scanning and mine detection, (2) The wooden arm.

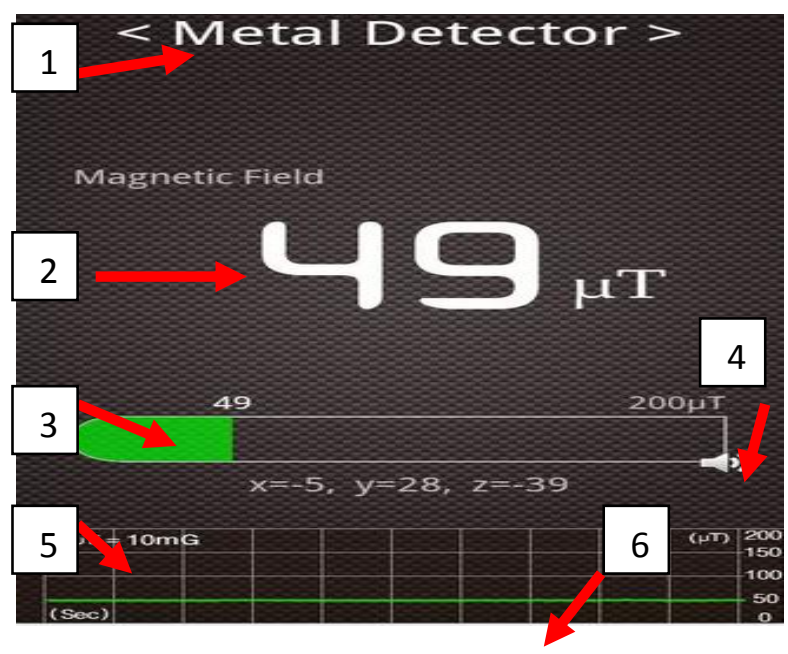

Fig 3: The metal detector's unite in a normal phase.(1) Metal detector app/Android operating system (OS), (2) Magnetic field level in nature is about $49 \mu \mathrm{T}$, (3) The green color reflects the natural state of magnetic field level, (4)

The sound alarm sign, (5) $1 \mu \mathrm{T}=10 \mathrm{mG}$, (6) The green color of line chart means that magnetic field level in natural range. 


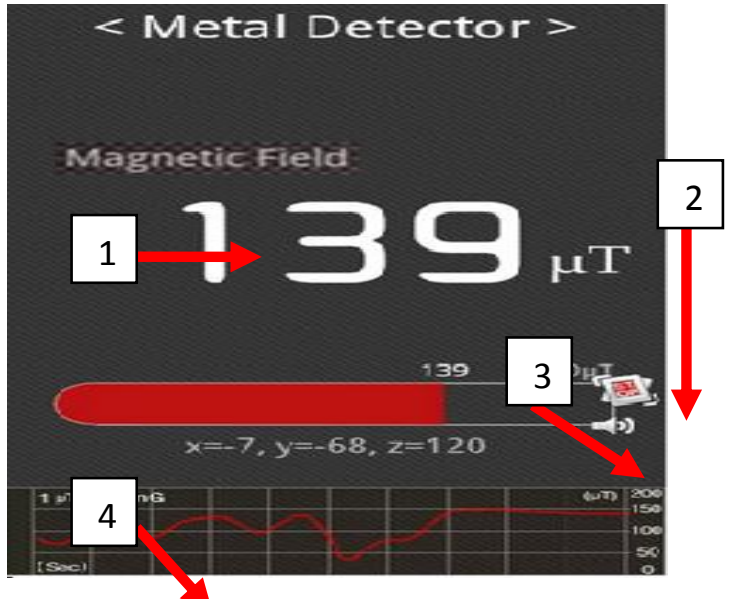

Fig 4: The metal detector detect a tiny metal nearby it. (1) Magnetic field up to a high level, (2) The cellphone vibrates due to the high magnetic field value, (3) A loudly alert beep occurs according to the same reason , (4) The red color of line chart means that magnetic field level is too high.

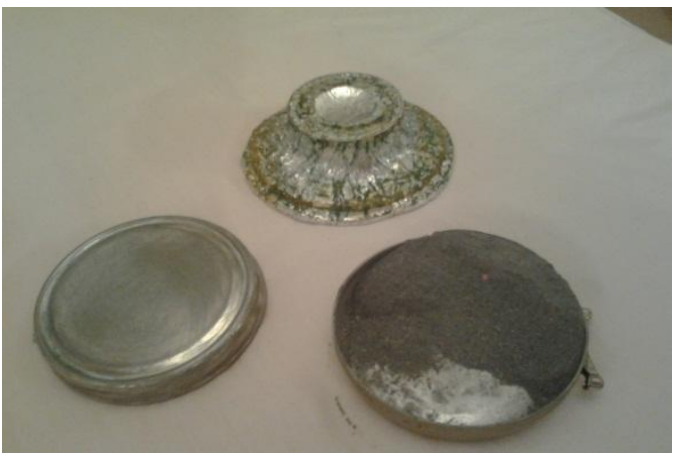

Fig 5: explosive capsule

\subsection{The explosives detection strategy}

As mentioned in section (2) the EDT is a mini RC truck, it is controlled by human to go all directions from a safe distance, so when the EDT go fare from the operator in a mine field how minor changes occur to the metal detector can be observed by the user from a distance when approaching or detecting a landmine?, In this paper the human audiovisual perception system were replaced by a GSM audio tracer device and GSM Surveillance camera that were attached to the EDT as shown in figure 6, these two devices work by prepaid SIM cards for each one of them to contribute followup changes felt by the HTC mine detector and transported directly to the user via mobile networks (3G base stations).

The GSM sound tracer device calls the operator from anywhere in the desert or mine field when it detects the loudly beep alert emitted by HTC mine detector when approaching a land mine, This process be workable when inserting a GSM SIM card into the GSM audio tracer device and send a SMS containing the activation word, followed by the user or the operator cell phone number from his mobile phone, the GSM audio tracer device is active and ready for calling back action, The GSM sound tracer device supported with an Ultra tiny dimension: 3 x 2.5 x $1.5(\mathrm{~cm})$, its Weight: $42 \mathrm{~g}$ only , the built in microphone range (Meter):5 7(Radius), Working Temp between $40^{\circ} \mathrm{C}$ to $50^{\circ} \mathrm{C}$ it is perfect for desert weather and for keeps the EDT's total weight in the limits of one kilogram. On the other side, by adjusting the angle of inclination to the
GSM surveillance camera to see the HTC metal detector screen to follow up any changes that occur to it, the GSM surveillance camera device works by SIM cards as the GSM sound tracer unit but the GSM surveillance camera provides real time video feed to the user's cellphone, the user can observes any minor changes occur to the HTC metal detector as shown in figure 7 , In other words the user can be see what GSM Surveillance camera sees at the same time.

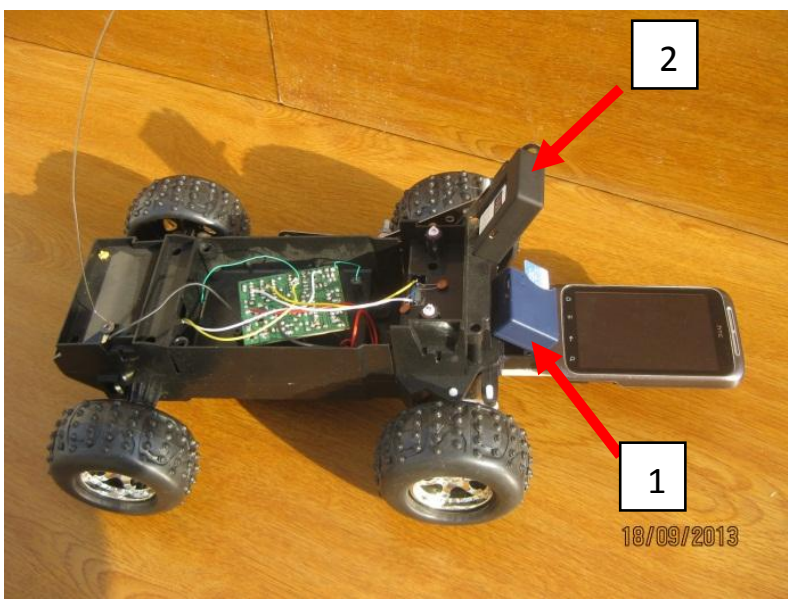

Fig 6: the human audiovisual perception system were replaced by a GSM audio tracer device and GSM Surveillance camera.(1) GSM audio tracer unit, (2) and GSM Surveillance camera.

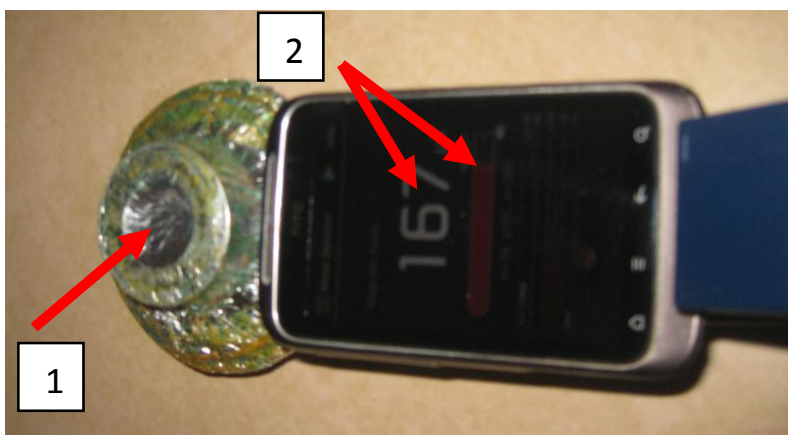

Figure 7: User can be see what GSM surveillance camera sees, the user can be observes any minor changes occur to the HTC metal detector when reach a land mine via his cellphone. (1) hand-made explosive capsule to simulates real land mine, (2) The HTC mine detector Felt the explosive capsule.

\subsection{The monitoring process and pinpoint the coordinates process strategies}

After confirming the presence of a land mine nearby the metal detector device through viewing and careful observation of the information which available by GSM audio tracker device and GSM surveillance camera, now the user can pinpoint the explosive coordinates by using the GPS tracker device that was attached to the EDT as shown in figure 8, it is a portable GPS tracking device with GSM base station locating \& GPS locating, it's provides web tracking and SMS tracking together, also the GPS tracker device works with a prepaid SIM card such as the rest devices, Whenever the user communicate directly with his mobile phone to this SIM card number in the tracker unit, the GPS tracer unit will respond with a text message containing the coordinates of where the EDT is located, user can use these coordinates on any 
mapping software (for example Google earth) which will show where exactly the land mine is, for example Copy and paste this coordinate into Google Earth $5129.5356^{\circ} \mathrm{N} 0$ 07.3159 W, will show how it pinpoints the Tracker.

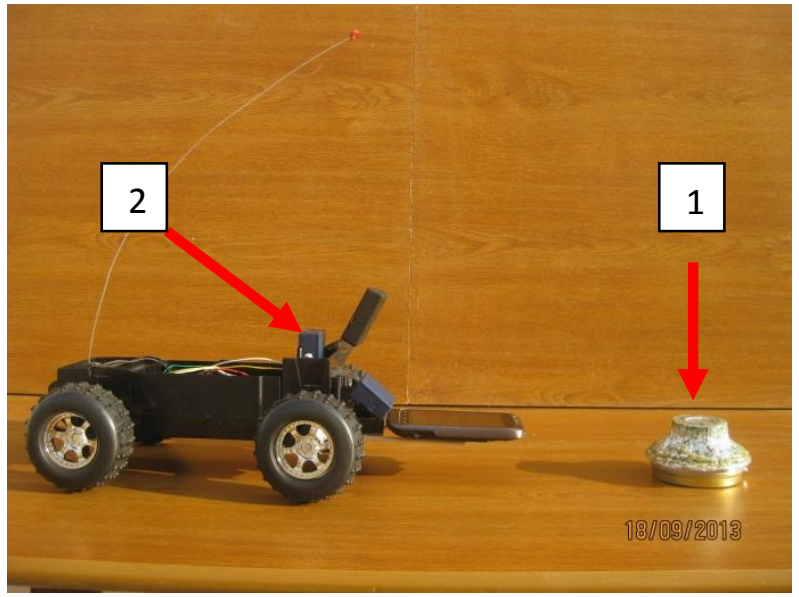

Fig 8: Pinpoint the location coordinates. (1)An explosive capsule is near by the mine detector, (2) The GPS tracker pinpoint the location coordinates for sending it to the user via SMS.

\section{CONCLUSION}

This paper presents a new technique for using metal detector in mine field from a distance in El Alamein region. The RC truck equipped with a wooden arm is to carry a sensitive cellphone without SIM card, plus Android OS and metal detector App to act as an advanced metal detector device. Another two sensitive devices attached to the truck to replace the human audiovisual perception, this units are the GSM audio tracer device and GSM surveillance camera, this two devices work by prepaid SIM cards for each one of them to contribute follow-up changes felt by the mine detector and communicate directly with user's cellphone via mobile networks and GPRS services such as short message and multimedia messaging to feed the user with minor changes that occur to the detector unit when approaching landmine. So the user can give an order by calling the SIM card number that inserted to the last tracking unit, it is the GPS tracker device, even the GPS unit can pinpoint the exact location of land mine and sending SMS to feedback the user with the coordinates so user can use these coordinates on any mapping software as Google earth which will show where exactly the land mine is.

\section{REFERENCES}

[1] A. S. Omar,rer. nat. Georg Rose and W. AlNuaimy,"GROUND PENETRATING RADAR SIGNAL PROCESSING FOR LANDMINE DETECTION",pp.1$135,2007$.

[2] Lundberg. M, "Electro-optical land mine detection and contributions to iterative decoding and power estimation", Technical report no. 3731., licenciate thesis, Department of Signals and Systems,School of Electrical and Computer Engineering, chalmers university of technology, Sweden, 2001..

[3] L. ROBLEDO*, M. CARRASCO and D. MERY, "A survey of land mine detection technology", International Journal of Remote Sensing, pp.1-9, 2008.
[4] N. B. Askar, R. Hamdan, M. El-Tarhuni, N. Qaddoumi, "A Wireless Anti-personal Landmine Detection System", 2nd International Conference on Modeling, Simulation, and Applied Optimization (ICMSAO'07), Abu Dhabi, March 2007.

[5] KOWALENKO, K." Saving lives, one land mine at a time",. The IEEE Institute, 28, 2004.

[6] HABIB, M.K., "Mine detection and sensing technologies - new development potentials in the context of humanitarian demining". Proceedings of the 27th Annual Conference of the IEEE Industrial Electronics Society, 3, pp. 1612-1621, 2001.

[7] RUSSEL, K.,Contact methods. In "Alternatives for Landmine Detection", J. MacDonald, J.R. Lockwood, J. McFee, T. Altshuler, T. Broach, L. Carin, R. Harmon, C. Rappaport, W. Scott and R. Weaber (Eds) (Pittsburg, PA: RAND) , 2003.

[8] GADER, P., "Pattern recognition for humanitarian demining". Proceedings of the 16th International Conference on Pattern Recognition, IEEE, 2, pp. 521522,2002 ..

[9] WEN-HSIUNG, L., GADER, P. and WILSON, J., "Optimizing the area under a receiver operating characteristic curve with application to landmine detection". IEEE Transactions on Geoscience and Remote Sensing, 45, pp. 389-397, 2007.

[10] NIEMAN, W., OLESINSKI, S., THIELE, T., MARTENS, G. and CARLSEN, I.C., "Detection of buried landmines with X-ray backscatter technology"., Insight, 44, pp. 634-636, 2002.

[11] Cheolha Pedro Lee, "Mine Detection Techniques Using Multiple Sensors", The Project in Lieu of Thesis, Electrical and Computer Engineering The University of Tennessee at Knoxville.

[12] Daniels, D., "GPR for landmine detection", an invited review paper, 10th International Conference on Ground Penetrating Radar, 21-24 June, Delft, The Netherlands, PIP.2, 2004 .

[13] MacDonald, J., Loockwood, J., Altshuler, T., Broach, T., Carin, L., Harmon, R., Rappaport, C., Scott, W., and Weaver, R., "Alternatives for landmine detection", RAND, USA, 336 pp., 2003..

[14] Cassinis, R., "Landmines Detection Methods Using Swarms of Simple Robots"., pp 1-7, 2000.

[15] Salinas, C., Armada, M., Gonzalez de Santos.,'DA new approach for terrain description in mobile robots for humanitarian demining mission.",Proceedings of the EURON/IARP International Workshop on Robotics for Riskt Interventions and Surveillance of the Environment, Benicassim, Spain, 2008..

[16] El-Shenawy,A., "The Construction of Autonomous Electric Vechle for Land mine Detection and Localization", IEEE, pp. 91-96, 2012..

[17] APOPO is a Belgian NGO, it is a social enterprise that researches, develops and implements detection rats technology for humanitarian purposes such as Mine Action and Tuberculosis detection.[http://www.apopo.org/en/about-apopo/aboutapopo/why-rats\#myModal31] Last visit at 5-2013. 